\title{
Chronic High-frequency Stimulation of the Subthalamic Nucleus Induces a Sustained Inhibition of Serotonergic System via Loss of Cell Phenotype
}

\section{Faisal Alosaimi}

Maastricht University Medical Centre

Yasin Temel

Maastricht University Medical Centre

Sarah Hescham

Maastricht University Medical Centre

Victoria Witzig

Universitätsklinikum Aachen

\section{Faris Almasabi}

Maastricht University Medical Centre

\section{Sonny Tan}

Universitätsklinikum Aachen

Ali Jahanshahi ( $\nabla$ a.jahanshahi@maastrichtuniversity.nl)

Maastricht University Medical Centre

\section{Research Article}

Keywords: deep brain stimulation, Parkinson's disease, neurotransmitter respecification, dorsal raphe nucleus

Posted Date: January 12th, 2022

DOI: https://doi.org/10.21203/rs.3.rs-1227919/v1

License: (c) (1) This work is licensed under a Creative Commons Attribution 4.0 International License. Read Full License 


\section{Abstract}

Deep brain stimulation (DBS) of the subthalamic nucleus (STN) has become a standard treatment in Parkinson's disease (PD). However, in a considerable number of patients debilitating psychiatric sideeffects occur. Recent research has revealed that external stimuli can alter the neurotransmitters' homeostasis in neurons, which is known as "neurotransmitter respecification". Herein, we addressed if neurotransmitter respecification could be a mechanism by which DBS suppresses the serotonergic function in the dorsal raphe nucleus (DRN) leading to mood changes. We infused transgenic 5-HT-Cre (ePet-cre) mice with AAV viruses to achieve targeted expression of eYFP and the genetically encoded calcium indicator GCaMP6s in the DRN prior to methyl-4phenyl-1,2,3,6-tetrahydropyridine (MPTP) treatment. Mice received bilateral DBS electrodes in the STN and an optic fiber in the DRN for Ca2+ photometry. MPTP treated mice demonstrated behavioral and histological PD phenotype, whereas all STN-DBS animals exhibited an increased immobility time in the forced swim test, reduced Ca2+ activity, and loss of TPH2 expression in the DRN. Given the prominent role of $\mathrm{Ca} 2+$ transients in mediating neurotransmitter respecification, these results suggest a chronic loss of serotonergic phenotype in the DRN following STN-DBS. These findings indicate that loss of 5-HT cell phenotype may underlie the unwanted depressive symptoms following STN-DBS.

\section{Introduction}

Deep brain stimulation (DBS) has emerged as a successful neurosurgical treatment to treat selected neurological and psychiatric disorders ${ }^{1-4}$. DBS of the subthalamic nucleus (STN) has particularly shown to effectively improve medically intractable motor symptoms of Parkinson's diseases (PD) ${ }^{5-8}$. In spite of long-term improvement in motor function, a number of PD patients exhibit mood disorders such as depression, suicide ideation and impulsivity after surgery 9,10.

Our earlier studies have shown that acute bilateral STN-DBS inhibits neurotransmission of the midbrain serotonin (5-hydroxytryptamine; $5-\mathrm{HT}$ ) system in the dorsal raphe nucleus (DRN), which is the main source of 5-HT in the central nervous system and its dysfunction has been associated with the onset of mood disorders ${ }^{11}$. Acute STN-DBS in experimental animal studies demonstrated reduced firing rate of DRN 5-HT neurons, decreased 5-HT release in the forebrain and induction of depressive-like behavior in PD rats ${ }^{12,13}$. However, in clinical settings STN-DBS is applied chronically. Long-term modulation of neuronal networks may induce permanent and neuroplastic changes ${ }^{14}$. More recently, it has been demonstrated that neurotransmitter identity in the mature brain can be influenced by environmental stimuli ${ }^{15}$. Neurotransmitter switching, induction or elimination associated with altered behavioral output are termed neurotransmitter respecification ${ }^{16-18}$. We hypothesized that neurotransmitter respecification plays a role in STN-DBS and occurs in the DRN 5-HT system. To investigate this we used the transgenic mouse line expressing Cre under the enhancer of the transcription factor Pet1 (ePET-Cre), which allows selective targeting of DRN 5-HT neurons ${ }^{19}$. These transgenic mice with PD associated symptoms after methyl-4phenyl-1,2,3,6-tetrahydropyridine (MPTP) administration were treated with chronic STN-DBS. 
Behavioral, photometric and immunohistochemical assessments were used to evaluate aspects of neurotransmitter respecification of the DRN 5-HT system.

\section{Results}

Stimulating electrodes were positioned bilaterally and symmetrically (inter-electrode variation $<0.1 \mathrm{~mm}$ ) in the STN in all mice except two, for which electrodes were located in the zona incerta. The latter mice were excluded from the analysis. Fiber photometry probes were placed in the dorsomedial segment of the DRN in all mice except three, which were excluded from signal processing. No signs of significant histological damage due to implantation or chronic stimulation were observed.

MPTP treated mice showed a PD-like motor phenotype. MPTP induced significant static and dynamic gait impairments with increased stance (MPTP-sham: $0.17 \pm 0.005$ versus $\mathrm{NaCl}$-sham: $0.13 \pm 0.004$; F $(1,51)=9.19, p<0.001)$, terminal dual stance (MPTP-sham: $0.02 \pm 0.002$ versus NaCl-sham: $0.13 \pm 0.001 ; F$ $(1,51)=2.9, p<0.001)$, step cycle (MPTP-sham: $0.3 \pm 0.008$ versus NaCl-sham: $0.25 \pm 0.007 ; F(1,52)=8.3$, $\mathrm{p}<0.001)$ and reduced average speed (MPTP-sham: $18.09 \pm 0.62$ versus $\mathrm{NaCl}$-sham: $22.83 \pm 0.83 ; \mathrm{F}$ $(1,52)=8.3, p<0.001)$ compared to NaCl-treated mice. STN-DBS restored these gait parameters in MPTPtreated mice with decrease stance (MPTP-stim: $0.12 \pm 0.004$ versus MPTP-sham: $0.17 \pm 0.005 ; \mathrm{F}$ $(1,51)=32.2, p<0.001)$, terminal dual stance (MPTP-stim: $0.009 \pm 0.001$ versus MPTP-sham: $0.02 \pm 0.002 ; F$ $(1,51)=19.59, p<0.001)$, step cycle (MPTP-stim: $0.24 \pm 0.009$ versus MPTP-sham: $0.3 \pm 0.008 ; F$ $(1,51)=19.63, p<0.001)$ and increased average speed (MPTP-stim: $23.9 \pm 0.81$ versus MPTP-sham: $18.09 \pm 0.62 ; F(1,52)=11.21, p<0.001)$ when they were compared to sham animals (Fig. 1A-D).

Stimulation did not alter gait parameters in $\mathrm{NaCl}$-treated mice. Post-mortem $\mathrm{TH}$ immunohistochemistry revealed a significant loss (60\%) of SNc dopaminergic neurons after MPTP administration in comparison to $\mathrm{NaCl}$ treatment (MPTP-sham: $198.8 \pm 30.54$ versus $\mathrm{NaCl}$-sham: $491.8 \pm 43.82$; independent samples Ttest $p<0.005$; Fig. 1E-G).

Fiber photometry assessing calcium signaling of DRN neurons showed a significant reduction of GCaMP6s fluorescence indicating neuronal inhibition upon STN-DBS (Fig. 2A-D). Permutation test showed decreased calcium signaling by STN-DBS in both MPTP and NaCl-treated mice $(p<0.05)$. After stimulation was halted GCaMP6s fluorescence signal returned to baseline within ninety seconds.

Interim analysis of FST showed no significant difference between groups $(F(1,27)=2.11$, p's $\geq 0.16$ Fig. S1). Therefore, the test was discontinued due to risk of drowning. The data of stimulated animals including both MPTP and NaCl-treated mice were pooled compared to the sham animals (stim: $139.56 \pm 14.39$ versus sham: $88.93 \pm 15.13$; independent samples T-test, $p<0.05$; Fig. $2 E$ ). STN-DBS induced behavioral despair in the FST, which was apparent by increased immobility time in comparison to nonstimulated mice. This depressive-like behavior after STN-DBS was observed in both MPTP and NaCltreated mice. 
After we established that STN-DBS induced depressive-like behavior and decreased calcium signaling in the DRN, we subsequently evaluated the phenotype of genetically targeted DRN 5-HT neurons. Stereological cell count of double-labelled eYFP/TPH2 expressing neurons in the DRN showed a significant decrease of double-labelled neurons in STN-DBS treated mice in comparison to sham stimulated animals (MPTP and NaCl-stim: $1670 \pm 144$ and 1590 \pm 141 , versus MPTP and NaCl-sham: $712 \pm 50$ and $518 \pm 83$, respectively (Fig. $2 F-H)$. This inhibitory effect of chronic STN-DBS on TPH2 expression was found to be independent of the integrity of the dopamine nigrostriatal pathway as this observation was present in both MPTP and NaCl-treated mice $(F(1,14)=0.25, p \geq 0.61$ Two-way ANOVA; Fig. 2F). Finally, STN-DBS nor MPTP administration altered neuronal c-Fos expression of the DRN. No significant changes between groups were found $(F(1,18)=0.001$ p's $\geq 0.97$ Fig. S2).

\section{Discussion}

In this study we have investigated neuroplastic effects of DBS on neurotransmitter phenotype. Recently, neurotransmitter respecification in the adult brain was described in which external cues induced neurotransmitter phenotype switching, neurotransmitter induction or elimination with concurrent behavioral alterations ${ }^{16-18}$. We hypothesized that this phenomenon may play a role in DBS. This may be particularly relevant for STN-DBS as a widely accepted neurosurgical treatment in medically refractory PD with stimulation dependent motor and non-motor behavioral changes ${ }^{5-8}$. Patients may experience depressive symptoms after surgery, which by itself is a risk factor for post-operative suicide 9,10 . Understanding the neuronal mechanisms of these behavioral changes is relevant for the more than 208,000 patients that are already treated by DBS worldwide ${ }^{20}$.

We used the ePET-Cre mouse line, which enables specific assessment of 5-HT neurons in the DRN synthesizing TPH ${ }^{19}$. MPTP administration in these mice resulted in a significant loss (approximately $60 \%$; Fig. 1E) of SNc dopamine neurons and displayed gait impairments that were alleviated by STN-DBS, overall mimicking dopaminergic degeneration and beneficial motor effects of stimulation in PD patients (Fig.1A-D).

In addition, STN-DBS elicited behavioral despair in MPTP mice, which reflects depressive-like behavior (Fig. 2E). This behavioral change by STN-DBS was independent of the integrity of the nigrostriatal pathway and motor function as $\mathrm{NaCl}$ - treated mice showed similar behavioral output (Fig. S1). This observation was also reported by our previous studies ${ }^{12,21}$. Pretreatment with the selective-serotonin reuptake inhibitor citalopram before STN-DBS was effective in preventing behavioral despair ${ }^{12}$. This pinpointed towards a 5-HT dependent mechanism and triggered experiments investigating downstream effects of STN-DBS to the brainstem 5-HT system, with the DRN as the major source of 5-HT innervation to the forebrain ${ }^{11}$.

Using fiber photometric measurements of calcium signaling we demonstrated in this study that chronic STN-DBS decreased calcium signaling and caused neuronal inhibition within the DRN (Fig. 2A-D). This is in line with acute STN-DBS electrophysiological experiments where stimulation decreased 5-HT neuronal 
firing rate by $40-50$ in extra-cellular single cell recordings ${ }^{12,22}$. Subsequent in vivo microdialysis experiments also found decreased $5-\mathrm{HT}$ release in terminal forebrain regions as expected ${ }^{13,23}$. Previous studies have focused on the underlying neuronal circuit. Since STN projecting neurons to the DRN are lacking it has been postulated that inhibition of 5-HT neurotransmission is mediated by a multi-synaptic neuronal network. The lateral habenula may contribute to this network as a well-defined major inhibitory input structure to the DRN and has been attributed a critical role in 5-HT feedback mechanisms 21,22 . Acute STN-DBS has altered neuronal firing rates of habenular neurons projecting to the DRN ${ }^{22}$. It remains undetermined how chronic STN-DBS influences 5-HT neurotransmission and homeostasis. It has been shown, however, that some cells regain the ability to fire intrinsic spikes of action potential at the presence of continuous stimulation ${ }^{24}$, whereas other neurons remain inhibited after cessation of stimulation ${ }^{22}$. These altered activities most likely influence stringent $5-\mathrm{HT}$ feedback mechanisms and may trigger neuroplasticity within the network.

Our earlier study indicated that DBS of the anterior nucleus of the thalamus increased the number of dopaminergic neurons in the ventral tegmental area ${ }^{25}$. This might have been indicative for DBS induced neurotransmitter respecification. In the current study eYFP positive neurons in the DRN should typically express TPH2 in the vast majority $(>90 \%)^{19}$. Interestingly, we found STN-DBS to reduce the number of double-labelled eYFP/TPH2 positive neurons quantified by stereological methods (Fig. 2F-H). Although ePET-Cre genetically targets DRN 5-HT neurons specifically, it should be kept in mind that it represents a part of the total $5-\mathrm{HT}$ population ${ }^{19}$. Moreover, $5-\mathrm{HT}$ cells only around the infusion site were transfected in this study. In addition, overall c-Fos expression in the DRN was not altered by STN-DBS, suggesting that overall neuronal activity after chronic stimulation remained stable (Fig. S1).

Activity dependent intracellular calcium transients play a key role in neurotransmitter respecification by regulating the phosphorylation of transcription factors that are critical in defining the neurotransmitter phenotype of cells $17,26,27$. However, how calcium transients alter neurotransmitter respecification, seems to differ across transmitter systems and species. For instance, elevated activity of dopaminergic neurons in the paraventricular nucleus of the hypothalamus in the adult rats was shown to be required for the loss of dopamine expression after long-day photoperiod exposure ${ }^{18}$. Whereas, decreases in calcium spiking by exposure of Xenopus laevis to dark lead to loss of dopamine expression in hypothalamus ${ }^{28}$. Seemingly, altered calcium transients could lead to opposite effects in serotonergic neurons. Suppression of activity in the Xenopus laevis hindbrain generated an increase in the number of neurons expressing TPH in the raphe nucleus. Whereas, enhancement of activity led to the opposite result ${ }^{27}$. In our study, a decrease in the number of TPH2 expressing neurons was associated with reduced $\mathrm{Ca} 2+$ transients. This is in contrast to respecification of dopaminergic cells in rats ${ }^{18}$, in which an increase in Ca2+ activity correlated with the loss of dopaminergic cell phenotype. Altogether these behavioral, photometric and immunohistochemical data pinpoint to a key role for stimulus-derived loss of 5-HT cell phenotype. We argue that this loss of 5-HT phenotype plays a key role in unwanted depressive symptoms following STNDBS. The fade of 5-HT phenotype could also be the mechanism whereby STN-DBS reduces treatment- 
resistant tardive dyskinesia. Ample evidence implicate the 5-HT system in the symptoms of dyskinesia. Extensive innervation of $5-\mathrm{HT}$ system to the basal ganglia modulates dopamine neurotransmission ${ }^{29,30}$. The lower incidence of dyskinesia associated with $5-\mathrm{HT} 2$ receptor antagonism ${ }^{31,32}$. Moreover, symptoms of dyskinesia can be exacerbated by concomitant treatment with selective serotonin reuptake inhibitors ${ }^{33-35}$. Based on our observation that STN-DBS suppress 5-HT cell phenotype, one may conclude that reduction in basal ganglia $5-\mathrm{HT}$ function is a key component of the DBS therapeutic mechanism in dyskinesia.

In conclusion, understanding neuroplastic effects is critical to our understanding of chronic network modulation by DBS and symptom reduction or side effects. This study reveals evidence that STN-DBS induces changes in calcium signaling in the midbrain raphe nuclei $5-\mathrm{HT}$ system and results in neurotransmitter respecification, which may play a role in psychiatric side effects in PD. The loss of 5-HT cell phenotype could also be the mechanism whereby STN-DBS reduces treatment-resistant tardive dyskinesia.

\section{Methods}

Animals

Experiments were performed on 56 male transgenic ePET-Cre mice (JAX stock; \#012712). Animals were socially housed under constant temperature, humidity and reversed dark/light cycle (12 hours each) with free access to food and water. All animal procedures were performed in accordance with "Animal Research: Reporting of In Vivo Experiments (ARRIVE)" guidelines. Animal procedures were reviewed and approved by the Institutional Animal Care Committee of Maastricht University in accordance to the Central Authority for Scientific Procedures on Animals (CCD; protocol \# AVD107002016543).

Induction of Parkinson's disease model and stereotactic surgery

Mice were randomly assigned into one of the following four groups: NaCl-sham, $\mathrm{NaCl}$ - STN-DBS, MPTPsham or MPTP-STN-DBS. MPTP was administered to induce dopaminergic neuron loss in the substantia nigra pars compacta (SNc). Mice were injected with MPTP (30 mg $/ \mathrm{Kg}$ i.p) or normal $\mathrm{NaCl}(0.9 \%$ i.p.) for five consecutive days, two weeks prior to stereotactic surgery. Stereotactic surgery ${ }^{36}$ was performed under isoflurane inhalation anesthesia (Abbott Laboratories; induction 4\%, maintenance 1.5-3\%) after analgetic pretreatment (buprenorphine, $0.1 \mathrm{mg} / \mathrm{Kg} \mathrm{s.C)}$ ). The mouse head was positioned and fixated in a stereotaxic frame (Stoelting). A body temperature of $37^{\circ} \mathrm{C}$ was maintained with a thermo-regulator pad. After local anesthesia (lidocaine 1\% s.c.) the skull was exposed and burr holes were made for implantation of bilateral STN electrodes (coordinates from bregma based on mouse brain atlas: AP $\left.-2.00 \mathrm{~mm}, \mathrm{ML} \pm 1.50 \mathrm{~mm}, \mathrm{DV}-4.55 \mathrm{~mm}^{37}\right)$ and a fiber photometry probe $(400 \mu \mathrm{m} ; 0.48 \mathrm{NA}$ Patchcord $)$ was implanted in the DRN (coordinates from bregma based on mouse brain atlas: AP -4.5, ML -0.25 , DV2.9 at a $32^{\circ}$ angle from the left). 
During the same surgery and before implantation took place, two viral vectors were injected into the DRN. A Cre-dependent adeno-associated virus encoding for enhanced yellow fluorescent protein (eYFP; AAV5.EF1a.DIO.eYFP.WPRE.hGH; Penn Vector Core, USA) was injected (1.0 $\mu \mathrm{l}$, at a rate of $0.1 \mu \mathrm{l} / \mathrm{min})$ into the DRN. In addition, an AAV vector ensuring targeted genetic encoding of the fluorescent Ca2+ indicator GCaMP6s (AAV5.Syn.Flex.GCaMP6s.WPRE.SV40; Addgene, USA) was also injected at the same coordinates (500nL; Nanoject I; Drummond Scientific).

Deep brain stimulation

After 2 weeks of recovery, chronic intermittent STN-DBS was performed for 10 weeks with 20 min stimulation sessions ( 5 times a week) with monophasic high frequency stimulation at $130 \mathrm{~Hz}$, a pulse width of $60 \mu$ s and a current intensity of $80 \mu \mathrm{A}$. Sham stimulated animals were connected but stimulation was omitted.

Fiber photometry

Ca2+ transients of DRN neurons was measured in MPTP and saline-treated mice using an established fiber photometry technique ${ }^{36}$. This method enable measuring the bulk Ca2+-dependent fluorescence of GCaMP6 during STN-DBS. A two-wavelength GCaMP fiber photometry system (Doric Lenses Inc., Quebec, Canada) was utilized for calcium signal recording. GCaMP and Ca2+-independent fluorescent signals were alternatingly excited by a $470 \mathrm{~nm}$ LED and a $405 \mathrm{~nm}$ LED (isosbestic reference signal), respectively. GCaMP6s fluorescence emissions were captured with a Newport 2151 Femtowatt Photoreceiver Module and the signals relayed into a Field Programmable Gate Array (FPGA)-based data acquisition unit which integrates with the Doric Neuroscience Studio software. During the photometry experiment, mice could move freely in their home cage. STN-DBS was applied intermittently ( 2 min on - 3 min off) for ten trials (5 min per trial) during which photometry measurements were performed during the DBS on/off phases. We extracted, processed and analyzed the calcium transients with a custom MATLAB (Mathworks) script. The first 2.5 minute of the data during the habituation period was discarded to remove the initial fast bleaching of the fluorescent signal. Next, the original sampling rate of a $100 \mathrm{~Hz}$ was downsampled to 1 $\mathrm{Hz}$ and low-pass filtered. A two-term exponential model was fitted and subtracted from the decimated data to account for slow bleaching artifacts. Then, a single baseline fluorescence value (F0) was calculated by averaging the fluorescent signals during the $60-\mathrm{sec}$ time period pre-DBS. Subsequently, the normalized change in fluorescence (dF/F) was calculated as F - F0/F0. Data are presented as an average plot with SEM. A permutation test was used to analyze the statistical significance of the DBS-related fluorescent change ${ }^{38}$. To compare the values of $\mathrm{dF} / \mathrm{F}$ at each time point with the DBS-related fluorescent change, 10,000 permutations were used. An a-level of $\leq 0.05$ was considered significant.

Behavioral assessment

- Gait analysis 
MPTP and STN-DBS related motor effects were assessed by a computerized gait analysis (CatWalkXT; Noldus), trained mice ran through an enclosed corridor with a hard glass plated floor. Footprints were recorded by a high speed camera from which gait-related movement parameters were analyzed, including average speed, stance, terminal dual stance and step cycle.

\section{- Forced Swim Test}

The forced swim test (FST) was used to evaluate despair behavior. Mice were placed in an inescapable plastic cylindrical container (height $40 \mathrm{~cm} x$ diameter $19 \mathrm{~cm}$ ) filled with a $23-25^{\circ} \mathrm{C}$ water $(30 \mathrm{~cm}$ deep). The duration of immobility was recorded during a trial of 6 minutes. Immobility was defined as the time of not moving or with slight movements to keep the nose above the water surface.

Tissue processing and immunohistochemistry

At the end of the experiments, mice were deeply anaesthetized with pentobarbital and transcardially perfused with tyrode buffer, followed by ice-cold $4 \%$ paraformaldehyde fixative in $0.1 \mathrm{M}$ phosphate buffer. The brains were extracted, fixed in $4 \%$ paraformaldehyde overnight and submerged in $20 \%$ sucrose for 24 hours at $5^{\circ} \mathrm{C}$. The brains were sectioned in coronal slices on a cryostat and stored at $-80^{\circ} \mathrm{C}$. A standard hematoxylin-eosin staining was performed to assess the electrode tip location. Animals with misplaced electrodes were excluded from behavioral and histological analysis.

- Tyrosine hydroxylase immunohistochemistry

MPTP induced SNc dopamine depletion was evaluated by tyrosine hydroxylase (TH) immunohistochemistry. Sections containing the SNc were incubated overnight with primary antibody raised against TH (rabbit polyclonal anti-TH antibody; Santa Cruz Biotechnology Inc; 1:1000). On the next day, sections were incubated with a secondary antibody (donkey anti-rabbit alexa 647, Jackson Immunoresearch Laboratories; 1:400) for one hour. Thereafter the sections were mounted and coverslipped (Immu-Mount, USA). Photographs of two anatomical bregma levels (coordinated based on mouse brain atlas AP -2.92 and -3.16 ${ }^{37}$ ) were taken with an Olympus DP70 digital camera connected to an Olympus BX50 microscope. A semi-quantitative TH cell count was performed using ImageJ software (National Institutes of Health, USA).

- c-Fos immunohistochemistry

To assess overall neuronal activity of the DRN immunohistochemical expression of the c-Fos was evaluated. DRN sections were incubated overnight with a primary anti-c-Fos antibody (rabbit polyclonal anti-c-Fos; Abcam; 1:1000). This was followed by incubation for one hour with a secondary antibody (donkey anti-rabbit alexa 594, Jackson immunoresearch Laboratory; 1:200). Eight slices were selected from bregma -4.16 to -4.96 and photographed with an Olympus BX51 fluorescence microscope (Olympus, Germany) connected to an Olympus Camera DP72 (Olympus, Germany). All clear c-Fos expressing neurons were counted (FiJi v2.0.0, National Institutes of Health; Maryland). 
To assess whether STN-DBS influenced 5-HT synthesis of eYFP expressing 5-HT DRN neurons, tissue was processed for tryptophan hydroxylase $2(\mathrm{TPH} 2)$ immunohistochemistry, which is the rate-limiting enzyme in 5-HT synthesis. DRN sections were incubated overnight with a primary anti-TPH2 antibody (goat polyclonal anti-TPH2; Abcam; 1:2000). This was followed by incubation with a secondary antibody (donkey anti-goat alexa 647, Jackson Immunoresearch Laboratories; 1:200) for two hours. Stereological analysis of double-labelled eYFP/TPH2 neurons was performed (Stereo Investigator, Microbrightfield Bioscience, Williston, VT, USA) in seven DRN sections per mouse using an immunofluorescence spinning disk confocal microscope (Olympus BX51, Japan) connected to a digital ultra-high sensitivity CCD camera (C9100-02, Hamamatsu Photonics, Japan). Stereological cell counting was performed using the optical fractionator and total double-labelled cell number was estimated using a validated stereological method $^{39,40}$.

Data analysis

Statistical analysis was performed using SPSS 26.0 software (SPSS Inc., Chicago, USA). Behavioral and immunohistochemical data were analyzed using the two-way ANOVA and LSD post-hoc comparison or independent T-test. Data are presented as mean values and standard error of means ( \pm SEM). Statistical significance was defined by a $p$-value $<0.05$. Photometry data was processed and analyzed with custom Matlab (MathWorks) scripts. A permutation test was performed to statistically evaluate calcium transients ${ }^{38}$.

\section{Declarations}

Acknowledgments

This work was funded by the Nederlandse Organisatie voor Wetenschappelijk Onderzoek (NWO-VENI) to AJ. FA thanks King Abdulaziz University for his PhD scholarship. The authors are tankful to Ms. Pol for her contribution to in vivo experiments.

\section{Author contributions}

AJ designed the study, AJ performed in vivo work, FA, VW, and FA (F. Almasabi) performed immunohistochemistry and microscopy work, AJ designed and adapted the program of the in vivo work, and supported management of the raw data, FA and AJ analyzed the data and wrote the manuscript, SH, ST and YT contributed to interpretation of the data, writing and editing the manuscript. All authors read and approved the final version of the manuscript.

Competing interests:

The authors declare no competing interests. 


\section{References}

1 Wichmann, T. \& DeLong, M. R. Deep brain stimulation for neurologic and neuropsychiatric disorders. Neuron 52, 197-204 (2006).

2 Benabid, A. et al. in Advances in Stereotactic and Functional Neurosurgery $10 \quad 39-44$ (Springer, 1993).

3 Andy, O. J. \& Jurko, F. Thalamic stimulation effects on reactive depression. Stereotactic and Functional Neurosurgery 50, 324-329 (1987).

4 Aouizerate, B. et al. Deep brain stimulation of the ventral caudate nucleus in the treatment of obsessive-compulsive disorder and major depression: Case report. Journal of neurosurgery 101, 682-686 (2004).

5 Deuschl, G. et al. A randomized trial of deep-brain stimulation for Parkinson's disease. New England Journal of Medicine 355, 896-908 (2006).

6 Group, D.-B. S. f. P. s. D. S. Deep-brain stimulation of the subthalamic nucleus or the pars interna of the globus pallidus in Parkinson's disease. New England Journal of Medicine 345, 956-963 (2001).

7 Limousin, P. et al. Electrical stimulation of the subthalamic nucleus in advanced Parkinson's disease. New England Journal of Medicine 339, 1105-1111 (1998).

8 Krack, P. et al. Five-year follow-up of bilateral stimulation of the subthalamic nucleus in advanced Parkinson's disease. New England Journal of Medicine 349, 1925-1934 (2003).

9 Voon, V., Kubu, C., Krack, P., Houeto, J. L. \& Troster, A. I. Deep brain stimulation: neuropsychological and neuropsychiatric issues. Mov Disord 21 Suppl 14, S305-327, doi:10.1002/mds.20963 (2006).

10 Bejjani, B. P. et al. Transient acute depression induced by high-frequency deep-brain stimulation. $N$ Engl J Med 340, 1476-1480, doi:10.1056/NEJM199905133401905 (1999).

11 Booij, L. et al. Tryptophan depletion affects heart rate variability and impulsivity in remitted depressed patients with a history of suicidal ideation. Biol Psychiatry 60, 507-514, doi:10.1016/j.biopsych.2006.02.010 (2006).

12 Temel, Y. et al. Inhibition of 5-HT neuron activity and induction of depressive-like behavior by highfrequency stimulation of the subthalamic nucleus. Proceedings of the National Academy of Sciences 104, 17087-17092 (2007).

13 Tan, S. K. et al. A combined in vivo neurochemical and electrophysiological analysis of the effect of high-frequency stimulation of the subthalamic nucleus on 5-HT transmission. Exp Neurol 233, 145-153, doi:10.1016/j.expneurol.2011.08.027 (2012). 
14 Jakobs, M., Fomenko, A., Lozano, A. M. \& Kiening, K. L. Cellular, molecular, and clinical mechanisms of action of deep brain stimulation-a systematic review on established indications and outlook on future developments. EMBO Mol Med 11, doi:10.15252/emmm.201809575 (2019).

15 Demarque, M. \& Spitzer, N. C. Neurotransmitter phenotype plasticity: an unexpected mechanism in the toolbox of network activity homeostasis. Developmental neurobiology 72, 22-32 (2012).

16 Dulcis, D., Jamshidi, P., Leutgeb, S. \& Spitzer, N. C. Neurotransmitter switching in the adult brain regulates behavior. Science 340, 449-453, doi:10.1126/science.1234152 (2013).

$17 \mathrm{Li}, \mathrm{H}$. Q. \& Spitzer, N. C. Exercise enhances motor skill learning by neurotransmitter switching in the adult midbrain. Nat Commun 11, 2195, doi:10.1038/s41467-020-16053-7 (2020).

18 Meng, D., Li, H. Q., Deisseroth, K., Leutgeb, S. \& Spitzer, N. C. Neuronal activity regulates neurotransmitter switching in the adult brain following light-induced stress. Proc Natl Acad Sci U S A 115, 5064-5071, doi:10.1073/pnas.1801598115 (2018).

19 Pinto, D. F. C. et al. Characterization of transgenic mouse models targeting neuromodulatory systems reveals organizational principles of the dorsal raphe. Nature communications 10, 1-14 (2019).

20 Vedam-Mai, V. et al. Proceedings of the Eighth Annual Deep Brain Stimulation Think Tank: Advances in Optogenetics, Ethical Issues Affecting DBS Research, Neuromodulatory Approaches for Depression, Adaptive Neurostimulation, and Emerging DBS Technologies. Front Hum Neurosci 15, 644593, doi:10.3389/fnhum.2021.644593 (2021).

21 Tan, S. K. et al. High frequency stimulation of the subthalamic nucleus increases c-fos immunoreactivity in the dorsal raphe nucleus and afferent brain regions. Journal of psychiatric research 45, 1307-1315 (2011).

22 Hartung, H., Tan, S. K., Temel, Y. \& Sharp, T. High-frequency stimulation of the subthalamic nucleus modulates neuronal activity in the lateral habenula nucleus. European Journal of Neuroscience 44, 26982707 (2016).

23 Navailles, S., Benazzouz, A., Bioulac, B., Gross, C. \& De Deurwaerdere, P. High-frequency stimulation of the subthalamic nucleus and L-3,4-dihydroxyphenylalanine inhibit in vivo serotonin release in the prefrontal cortex and hippocampus in a rat model of Parkinson's disease. J Neurosci 30, 2356-2364, doi:10.1523/JNEUROSCI.5031-09.2010 (2010).

24 Ammari, R., Bioulac, B., Garcia, L. \& Hammond, C. The Subthalamic Nucleus becomes a Generator of Bursts in the Dopamine-Depleted State. Its High Frequency Stimulation Dramatically Weakens Transmission to the Globus Pallidus. Front Syst Neurosci 5, 43, doi:10.3389/fnsys.2011.00043 (2011).

25 Dela Cruz, J. A. et al. Increased number of TH-immunoreactive cells in the ventral tegmental area after deep brain stimulation of the anterior nucleus of the thalamus. Brain Struct Funct, 
doi:10.1007/s00429-014-0832-7 (2014).

26 Spitzer, N. C. Activity-dependent neurotransmitter respecification. Nat Rev Neurosci 13, 94-106, doi:10.1038/nrn3154 (2012).

27 Demarque, M. \& Spitzer, N. C. Activity-dependent expression of $L m \times 1 b$ regulates specification of serotonergic neurons modulating swimming behavior. Neuron 67, 321-334, doi:10.1016/j.neuron.2010.06.006 (2010).

28 Dulcis, D. \& Spitzer, N. C. Illumination controls differentiation of dopamine neurons regulating behaviour. Nature 456, 195-201, doi:10.1038/nature07569 (2008).

29 Alex, K. D. \& Pehek, E. A. Pharmacologic mechanisms of serotonergic regulation of dopamine neurotransmission. Pharmacol Ther 113, 296-320, doi:10.1016/j.pharmthera.2006.08.004 (2007).

30 Di Matteo, V. et al. Serotonin modulation of the basal ganglia circuitry: therapeutic implication for Parkinson's disease and other motor disorders. Prog Brain Res 172, 423-463, doi:10.1016/S00796123(08)00921-7 (2008).

31 Kapur, S., Zipursky, R. B. \& Remington, G. Clinical and theoretical implications of 5-HT2 and D2 receptor occupancy of clozapine, risperidone, and olanzapine in schizophrenia. Am J Psychiatry 156, 286293, doi:10.1176/ajp.156.2.286 (1999).

32 Creed-Carson, M., Oraha, A. \& Nobrega, J. N. Effects of 5-HT(2A) and 5-HT(2C) receptor antagonists on acute and chronic dyskinetic effects induced by haloperidol in rats. Behav Brain Res 219, 273-279, doi:10.1016/j.bbr.2011.01.025 (2011).

33 Caley, C. F. Extrapyramidal reactions from concurrent SSRI and atypical antipsychotic use. Can J Psychiatry 43, 307-308 (1998).

34 Lauterbach, E. C. \& Shillcutt, S. D. Dyskinesia with fluoxetine: tardive or late-onset persistent, acute norfluoxetine dyskinesia? J Clin Psychiatry 58, 85-86 (1997).

35 Sandler, N. H. Tardive dyskinesia associated with fluoxetine. J Clin Psychiatry 57, 91 (1996).

36 Pol, S., Temel, Y. \& Jahanshahi, A. A Custom Made Electrode Construct and Reliable Implantation Method That Allows for Long-Term Bilateral Deep Brain Stimulation in Mice. Neuromodulation 24, 212219, doi:10.1111/ner.13165 (2021).

37 Fraklin, K. B. J. \& Paxinos, G. The Mouse Brain in Stereotaxic Coordinates Third Edition. Academic Press Third (2007).

38 Jean-Richard-Dit-Bressel, P., Clifford, C. W. G. \& McNally, G. P. Analyzing Event-Related Transients: Confidence Intervals, Permutation Tests, and Consecutive Thresholds. Front Mol Neurosci 13, 14, 
doi:10.3389/fnmol.2020.00014 (2020).

39 West, M., Slomianka, L. \& Gundersen, H. J. G. Unbiased stereological estimation of the total number of neurons in the subdivisions of the rat hippocampus using the optical fractionator. The Anatomical Record 231, 482-497 (1991).

40 Schmitz, C. \& Hof, P. R. Recommendations for straightforward and rigorous methods of counting neurons based on a computer simulation approach. Journal of chemical neuroanatomy 20, 93-114 (2000).

\section{Figures}
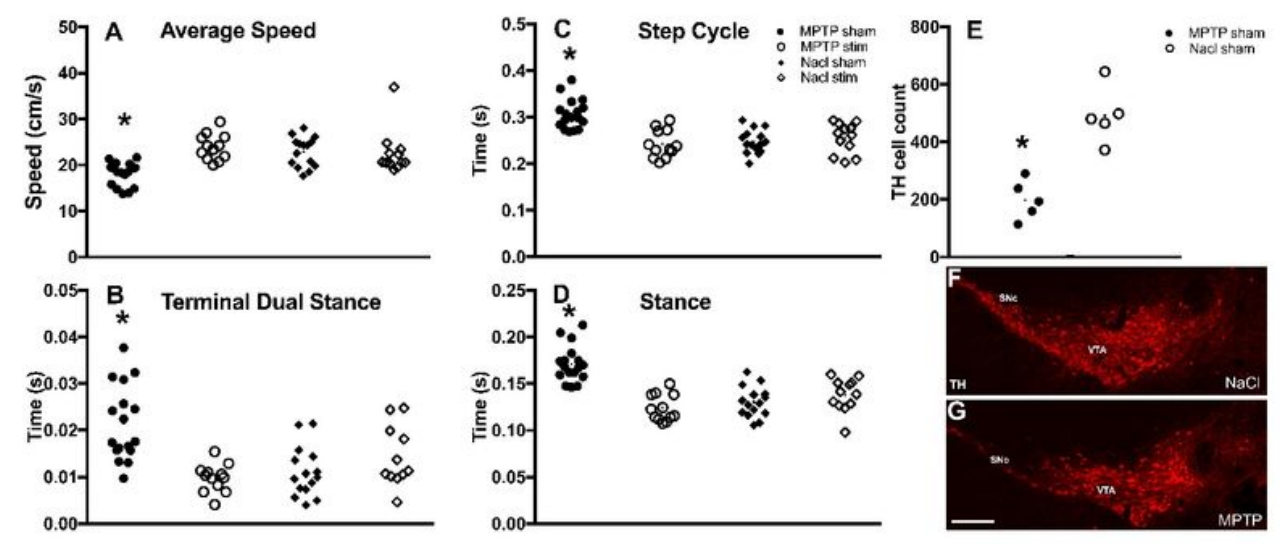

\section{Figure 1}

Effect of MPTP treatment and chronic STN-DBS on Catwalk dynamic and static gait parameters. A-D) Graphs show a significant reduction in speed, and increases of step cycle, terminal dual stance and stance in MPTP-sham mice. STN-DBS restored those parameters to control levels, which is indicated by non-significant differences between MPTP-stim and NaCl-sham groups. E) The graph shows a significant reduction in TH positive cells in the SNc of MPTP-treated mice compared to the NaCl-treated animals. FG) Representative low-power photomicrograph of coronal brain sections containing the SNc and VTA, stained for TH, show a noticeable TH cell loss in MPTP versus NaCl-treated mice. Data are presented mean $+/$ - SEM; significant difference $(P<0.05)$ is indicated by a " ", scale bar $=250 \mu \mathrm{m}$. Tyrosine hydroxylase, TH; substantia nigra pars-compacta, SNc; ventral tegmental area, VTA; subthalamic nucleus, STN; methyl-4phenyl-1,2,3,6-tetrahydropyridine, MPTP. 

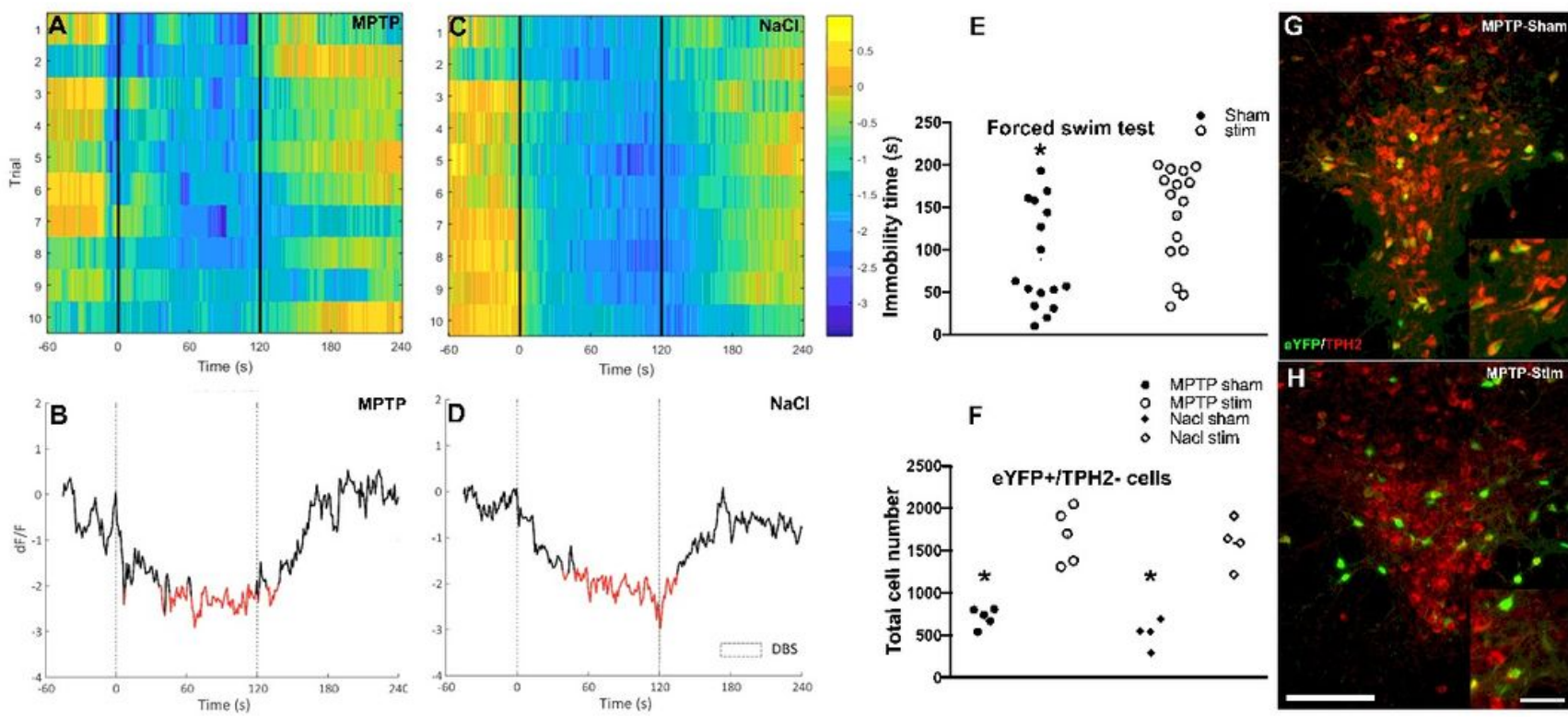

\section{Figure 2}

Effect of Chronic STN-DBS on serotonergic system. The effect of STN-DBS on activity of 5-HT neurons in the DRN measured with genetically coded calcium sensor GCaMP6s (fiber photometry). A and C) examples of heat-maps of the change in fluorescence (dF/F) before, during (between the dotted lines), and after DBS in MPTP and NaCl-treated mice, respectively. Each row plots one DBS trial (total of 10). Color scale at the right indicates $\mathrm{dF} / \mathrm{F}$ (yellow $=$ high and dark blue $=$ low dF/F). B and D) the bottom plots show the cumulative changes in fluorescence averaged over the ten trials in MPTP- $(n=14)$ and $\mathrm{NaCl}-$ treated mice $(n=17)$. The thick black line indicates mean, shaded areas indicate SEM, and red segments indicate statistically significant decrease from baseline ( $p<0.05$; permutation test). E) STN-DBS induced depressive like-behavior in forced swim test, shown by an increased immobility time in stimulated animals. F) The graph shows that chronic STN-DBS significantly reduced TPH2 expression in transfected (eYFP expressing) cells in both MPTP- and NaCl-treated mice. G-H) Representative photomicrographs of coronal brain sections containing the DRN display eYFP expressing cells that were double labelled with antibody raised against TPH2 (Scale bar $=150 \mu \mathrm{m}$ ). Insets in G-H show higher magnification of eYFP cells that with and without TPH-2 labeling (Scale bar $=50 \mu \mathrm{m}$ ). Data are presented mean $+/-\mathrm{SEM}$; significant difference $(P<0.05)$ is indicated by a "*". Subthalamic nucleus, STN, dorsal raphe nucleus, DRN, Enhanced yellow fluorescent protein, eYFP; methyl-4phenyl-1,2,3,6-tetrahydropyridine, MPTP.

\section{Supplementary Files}

This is a list of supplementary files associated with this preprint. Click to download.

- Alosaimietal2022Supplementarymaterial.docx 
- AlosaimietalFigureS2.tif

- AlosaimietalFigureS1.tif 